aged 68. This association was not attenuated when adjusting for childhood and adult socio-economic position.

Conclusion These findings show the lifelong importance of parenting on psychological self-concepts. Restricting young-life psychological freedom may build an enduring feeling that one is not agent of one's own control. Despite having access to a comfortable socio-economic position, those whose parents were more controlling in adolescence felt less in control of their own life age 68 .

\section{P12 EVALUATING POLICY RESPONSES TO UPSTREAM DETERMINANTS OF CHRONIC, NON-COMMUNICABLE DISEASES: SUPPORTING HEALTHY DIETS AND ACTIVE LIVING IN SEVEN CARIBBEAN COUNTRIES}

\begin{abstract}
${ }^{1}$ MM Murphy* ${ }^{2,3} \mathrm{C}$ Guell, ${ }^{1,4} \mathrm{TA}$ Samuels, ${ }^{1} \mathrm{~L}$ Bishop, ${ }^{3,4} \mathrm{~N}$ Unwin. ${ }^{1}$ Faculty of Medical Sciences, University of the West Indies, Bridgetown, Barbados; ${ }^{2}$ European Centre for Environment and Human Health, University of Exeter Medical School, Truro, UK; ${ }^{3} M R C$ Epidemiology Unit and Centre for Diet and Activity Research, University of Cambridge, Cambridge, UK; ${ }^{4}$ Chronic Disease Research Centre, University of the West Indies, Bridgetown, Barbados
\end{abstract}

\subsection{6/jech-2017-SSMAbstracts. 114}

Background In 2007, heads of government in the Caribbean Community (CARICOM) committed to concerted policy action to address non-communicable diseases, whose burden was recognised as a threat to regional development. In 2015, a large mixed-method evaluation study investigated the progress made in developing and implementing relevant policies. As part of this, a qualitative study in seven Caribbean countries aimed to identify, assess and compare existing policies, gaps in policy responses, and the factors influencing successful policy development and implementation.

Methods Policy document analysis was complemented by 76 semi-structured interviews with 80 relevant stakeholders in government, civil society and the private sector. Data collection and analysis protocols were developed iteratively. Interviews were audio-recorded and analysed pragmatically framed by the WHO NCD Action Plan, a Multiple Streams policy approach and realist evaluation principles. An analysis team coded using Dedoose software, after which two lead researchers synthesised the analyses.

Results Policy gaps existed regarding alcohol, diet and physical activity. Most widely reported successes across countries were policies and health promotion initiatives to support healthy eating in communities and in schools, including the development of dietary guidelines. Physical activity was targeted primarily in schools, with public participation in public sports events. Successful initiatives were often marked by collaboration between government ministries such as health, education and agriculture. There were very few existing policies around alcohol harm. The impact of these initiatives was reported as limited by adverse upstream determinants, including reliance on food imports entwined with the consequences of upholding existing trade agreements, which constrained more impactful fiscal and legislative action to support availability, quality and affordability of healthy foods. There was little evidence of policy responses to create physical and social environments conducive to active living, such as to support greater walking. The lack of focus on policy creation, lack of monitoring and evaluation of existing interventions, and limited staffing and financial capacities limited success in policy implementation. For alcohol specifically, national and political cultural attitudes and its economic importance deemed it untouchable from a policy standpoint.

Conclusion The least well developed policy responses concern upstream determinants of unhealthy diets, physical inactivity and alcohol harm. Local and regional political support is essential to accelerate action to support environments conducive to healthy eating and active living. Addressing reliance on food imports and existing trade agreements as well as developing and implementing policies to reduce alcohol related harm necessitates regional cooperation for a unified response.

\section{P13 SOCIAL, BIOLOGICAL, BEHAVIOURAL AND PSYCHOLOGICAL FACTORS AND PHYSICAL ACTIVITY DURING PREGNANCY: A CROSS-SECTIONAL STUDY}

${ }^{1} \mathrm{C}$ Flannery*, ${ }^{2} \mathrm{D}$ Dahly, ${ }^{1} \mathrm{M}$ Byrne, ${ }^{3}$ Ali Khashan, ${ }^{3} \mathrm{~S}$ McHugh, ${ }^{4} \mathrm{~L}$ Kenny, ${ }^{5} \mathrm{FM}$ McAuliffe, ${ }^{3} \mathrm{PM}$ Kearney. ${ }^{1}$ Health behaviour change research group, National University of Ireland, Galway, Ireland; ${ }^{2}$ HRB Clinical Research Facility Cork, University College Cork, Cork, Ireland; ${ }^{3}$ Department Epidemiology and Public Health, University College Cork, Ireland; ${ }^{4}$ Department Obstetrics and Gynaecology, University College Cork, Ireland; ${ }^{5}$ Obstetrics and Gynaecology, School of Medicine, University College Dublin, Ireland

\subsection{6/jech-2017-SSMAbstracts. 115}

Background Regular Physical Activity (PA) during pregnancy is associated with a number of health benefits such as preventing pregnancy complications, limiting pregnancy weight gain, and decreasing the risk of gestational diabetes. Despite this, women's PA levels often reduce or cease during pregnancy. Various factors like body mass index, unemployment, and educational levels have been shown to be associated with PA levels during pregnancy. Using the Screening for Pregnancy Endpoint (SCOPE) data, this study aimed to examine the association of health behaviours and psychological well-being on PA levels in pregnancy using the biopsychosocial model.

Methods Nulliparous women with a singleton pregnancy were recruited from a large academic maternity hospital in Cork, Ireland $(\mathrm{CUMH})$ as part of the international SCOPE study. Data was collected at $15 \pm 1$ week's gestation including personal information, lifestyle and psychological measures. The outcome of interest was PA categorised as low, moderate or high levels and covariates were selected using the biopsychosocial model including social (age; years of schooling; socioeconomic status), biological (body mass index), behavioural (diet; smoking) and psychological factors (anxiety; response to pregnancy). Univariate and multivariable multinomial logistic regression examined the association between covariates and PA using Stata IC13.

Results 1774 pregnant women were recruited to SCOPE and women were classified as having low (22.3\%), moderate $(54.4 \%)$ or high $(23.4 \%)$ PA levels $(n=1766)$. In the fully adjusted model, preliminary findings indicate that age categories 30-34 years (OR 2.34 [95\% CI: 1.26-4.37]), $\geq 35$ years (OR 2.06 [95\% CI: 1.01-4.19]) compared to those $<25$ years were positively associated with high PA levels. Having more than 12 years of schooling and a higher socioeconomic status remained significant $(\mathrm{p}<0.05)$ for moderate PA levels. Five portions of fruit and veg a day (OR 1.89 [95\% CI: 1.212.94]), fish consumption (OR 1.47 [95\% CI: 1.07-2.03]) and psychological response to pregnancy (OR 1.04 [95\% CI: 1.01-1.08]) were also positively associated with high levels of PA. Women who reported smoking in their first trimester were $31 \%$ less likely to be in the high physical activity group compared to those who reported no smoking (OR $0.69[95 \%$ 
CI: 0.49-0.99]). None of the biological factors were associated with high levels of PA.

Conclusion Social and behavioural factors appear to be the most influential factors driving PA levels. The results should be used to guide the development of educational campaigns and behaviour change intervention to encourage younger women to be active and to target those with lower education attainment and of lower socioeconomic status.

\section{P14 ASSOCIATION OF SLEEP DURATION AND SLEEP QUALITY WITH THE LOSS OF FUNCTIONING AMONG AUSTRALIAN PEOPLE}

\begin{abstract}
${ }^{1,2}{ }^{2}$ L Lallukka*, ${ }^{3,4,9} \mathrm{BS}$ Sivertsen , ${ }^{1}$ EK Kronholm, ${ }^{5} \mathrm{YSB}$ Bin, ${ }^{6,7}$ SØ Øverland, ${ }^{8} \mathrm{NG}$ Glozier, ${ }^{1} \mathrm{JHH}$ Halonen. ${ }^{1}$ Research and Service Centre for Work Organisations, Finnish Institute of Occupational Health, Helsinki and Turku, Finland; '2Department of Public Health, University of Helsinki, Helsinki, Helsinki; ${ }^{3}$ Department of Health Promotion, Norwegian Institute of Public Health, Bergen, Norway; ${ }^{4}$ The Regional Centre for Child and Youth Mental Health and Child Welfare, Uni Research Health, Bergen, Norway; ${ }^{5}$ Central Clinical School, Sydney Medical School, University of Sydney, Sydney, Australia; ${ }^{6}$ Norwegian Institute of Public Health, Bergen, Norway; ' Department of Psychosocial Science, University of Bergen, Bergen, Bergen; ${ }^{8}$ Brain and Mind Centre, University of Sydney, Sydney, Australia; ${ }^{9}$ Department of Research and Innovation, Helse Fonna HF, Haugesund, Norway
\end{abstract}

\subsection{6/jech-2017-SSMAbstracts.116}

Background While short and long sleep duration and poor sleep quality have individually been linked to work disability and ill-health, their joint impact on functioning has received less attention. The aim was to examine the joint associations of sleep quantity and quality with physical, emotional, and social functioning, while considering the contribution of key potential confounders.

Methods Data from the nationally representative Household, Income and Labour Dynamics in Australia (HILDA) Study, collected in 2013, were used $(n=14557)$. Poor sleep quality (yes/ no) was examined in combination with normal (6-8 hours), short $(<6)$ or long $(>8)$ sleep. Short form SF-12 questionnaire was used to examine functioning. Covariates included sociodemographic factors, social connexions (meeting with friends), health behaviours, obesity, pain, and mental and physical illnesses. Logistic regression analysis was used (odd ratios, OR and their 95\% confidence intervals, 95\% CI).

Results After adjusting for sex and age, short, normal and long sleep duration in combination with poor quality were all associated with poorer physical, emotional and social functioning. The associations were relatively similar for physical and emotional functioning, and strongest for social functioning (OR 3.36, 95\% CI 2.94-3.84 for normal sleep with poor sleep quality, OR 4.56, 95\% CI 4.02-5.17 for short sleep with poor sleep quality and OR 7.83, CI 95\% 6.09-10.09 for long sleep with poor sleep quality). Also people with good sleep quality but short or long sleep had poorer functioning, but the associations were weaker for all forms of functioning (ORs ranging 1.28-1.57 after adjusting for sex and age). Pain and comorbid illness attenuated all of the associations the most, while the contributions of the other covariates were relatively minor. However, the associations remained after full adjustments for people with poor sleep quality and short, normal or long sleep, and for people with good sleep quality but long sleep duration.
Conclusion The results highlight that in particular poor sleep quality is likely to have the most severe consequences for daytime functioning in the general population, and that there is little difference between short and normal sleepers in the presence of poor quality sleep.

\section{P15 INEQUALITIES IN ALCOHOL-RELATED MORTALITY: AN ELECTRONIC COHORT STUDY OF INTERNAL MIGRATION IN WALES}

A Gartner*, D Farewell, G Greene, L Trefan, S Paranjothy, D Fone. Division of Population Medicine, School of Medicine, Cardiff University

\subsection{6/jech-2017-SSMAbstracts. 117}

Introduction Alcohol-related deaths show a strong socioeconomic gradient. Selective migration, when individuals in poorer health move to more deprived areas, or vice versa, is associated with widening health inequality. However, it is not known whether recent internal migration contributes to the observed health inequality in alcohol-related deaths. This study aims to investigate whether selective migration of individuals is associated with the alcohol-related mortality gradient.

Methods We constructed a record-linked electronic cohort of the total population of Wales registered with a GP on 01/01/ 2006 ( $n=3$ 136881) with six-years' follow-up to 31/12/2011 in the SAIL Databank at Farr@Swansea. The Welsh Demographic dataset provided information on age, sex, and the lower super output area (LSOA) $(n=1896)$ of residence at each of the 24 quarters, used to code migration. To each LSOA code we linked tenths of the Welsh Index of Multiple Deprivation 2008 and the ONS Rural-Urban definition. To code alcohol-related mortality ( $n=2224$ of 184247 deaths) using the standard ONS definition we linked the demographic dataset to the ONS mortality files.

We estimated hazard ratios (HR) with 95\% confidence intervals $(95 \% \mathrm{CI})$ using Cox regression for the risk of alcohol-related death for deprivation fifths, adjusting for agegroup, sex and rural-urban status. The first model estimated HRs allowing for changing deprivation fifths linked to each move and the second model fixed the deprivation fifth at the start of the study period. A third model considered deprivation change between high (the five most deprived tenths) and low deprivation (the five least deprived tenths).

Results We found 495 subjects moved between LSOAs prior to an alcohol-related death. Of these, the majority (260) moved between high deprivation LSOAs and 75 moved between low deprivation LSOAs. Slightly more subjects moved from areas of low to high deprivation (98) than the opposite (62).

In model 1 subjects living in the most deprived fifth had a higher risk of alcohol-related death (HR 3.20; 95\% CI 2.77 to 3.71) compared to subjects in the least deprived fifth. In model 2 the risk was little different (HR 2.96; 95\% CI 2.56 to 3.42). Formal statistical adjustment for recent migration had little effect on the inequality estimates. In model 3, there was an elevated risk of alcohol-related death for moving from low to high deprivation.

Conclusion Internal migration within Wales over a six-year period does not explain the observed inequality in alcoholrelated mortality. 